\title{
Comparative Study on Wettability of Typical Plant Leaves and Biomimetic Preparation of Superhydrophobic Surface of Aluminum Alloy
}

\author{
Yanhong $\mathrm{Sun}^{1}$, Xinxin $\mathrm{Gao}^{1}$, Jinnong $\mathrm{Li}^{1}$, and Ying Chen ${ }^{1 *}$ \\ ${ }^{1}$ Jilin Engineering Normal University, College of Mechanical Engineering, 130052 Changchun, China
}

\begin{abstract}
The water droplets spread observation, contact angle measurement and SEM analysis, data processing and other means, comparative study of different states of the lotus leaf, rice leaf and nasturtium leaf are carried out. The results show that their surface contact angle of three kinds of plant leaves are all over $140^{\circ}$, they have hydrophobic or superhydrophobic properties; dehydration degree of leaves has little effect on the wettability, will not change its hydrophobic property; the same point of wettability is that they have micro nano composite microstructure. The difference is, the wettability of the lotus and nasturtium is isotropic, while the rice is anisotropic, that is, in a direction parallel to the edge of hydrophobic than perpendicular to the blade edge enhancement. Based on the theoretical research, the hydrophobic surface is copied to the surface of aluminum alloy. The superhydrophobic surface of aluminum alloy matrix was successfully prepared by chemical etching combined with surface fluorination. The super hydrophobic aluminum alloy surface has micron-nano scale double roughness structure; its surface contact angle reached $156^{\circ}$.
\end{abstract}

\section{Introduction}

After billions of years of evolution, natural creatures have acquired near perfect structure and strong environmental adaptability [1]. When the water drops on the lotus leaf surface, not spreads out, but close to the perfect spherical rolling on the lotus leaf surface, falling free, "Pearls falling into a jade plate" fantastic, as shown in Fig. 1. The water drops from the lotus leaves can carry away the dust, so the lotus leaves have "self-cleaning" characteristics. In 1977, William Barthlott first named the self-cleaning phenomenon known as the "lotus leaf effect" (Lotus- effect)" [2]. It contains profound scientific principles - "Infiltrative" [3,4]. It is governed by the laws of science. If the contact angle of water drop on solid surface is greater than $150^{\circ}$, it is called super hydrophobic surface [5], lotus leaves are super hydrophobic.

Although experts and scholars have made many achievements on superhydrophobic surface of plants. But, not to carry out a comparative study on the hydrophobic mechanism of typical plants, research on the causes of the difference in wetting function of typical

* Corresponding author: 823068684@qq.com 
plants. The superhydrophobic mechanism and the similarities and differences of lotus leaf, rice and nasturtium are not reported.

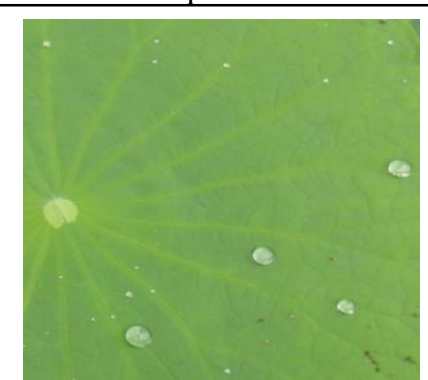

(a)Fresh lotus leaf

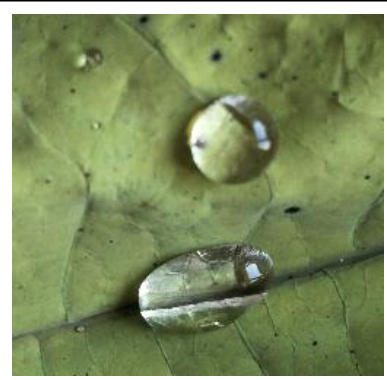

(b) Dried lotus leaf

Fig. 1. Drops of water on lotus leaves

\section{Equipment}

Using S-300N (Japanese manufacturing HITACHI) SEM microstructure and morphology analysis of plant leaf surface observation; Application of DSA100 surface analysis system (German manufacturing company of Kruss) and water contact angle measurement of the sample surface, water volume is $5 \mathrm{~L}$.

\section{Wettability contrast experiment for typical plants of different dehydrated leaves}

Fresh plant leaves are picked up after the source of moisture is cut off. As time goes on, the surface moisture decreases gradually. The influence of dehydration degree on the wettability of leaves was investigated. Through the direct observation of the distribution of water droplets on the blade, contact angle measurement and microscopic analysis, the wettability of the three samples was compared.

\subsection{Lotus leaf}

Lotus is a large perennial herb. It is long rhizome, leaves come directly from the root, long stalked, spiny, blade peltate, projecting above water[1].

As shown Fig. 1, the difference of surface wettability of between the fresh and the dried lotus leaves was observed. For the fresh leaf, the water droplets together, forming a large drop of water, and can roll freely, showed a strong hydrophobic function; For the dried leaf, little drops by two and three everywhere, the hydrophobic property is not inferior to the fresh leaves. Therefore, lotus leaves showed hydrophobic characteristics in two kinds of state. The contact angle of lotus leaves is shown in Table 1.

Table 1. Measurement results of contact angles of lotus leaves

\begin{tabular}{|c|c|}
\hline Types and locations of measurements & Contact angle ${ }^{\circ}{ }^{\circ}$ \\
\hline Fresh lotus leaf & $151 \pm 3$ \\
\hline Dried lotus leaf & $155 \pm 3$ \\
\hline
\end{tabular}

The Fig. 2 shows the morphology of lotus leaf surface. Its structure is composed of a large number of evenly distributed micro hemispherical papillae of about $10 \sim 15 \mu \mathrm{m}$ diameter, 2-5 $\mu \mathrm{m}$ height, $10 \sim 20 \mu \mathrm{m}$ distance, which are covered with $200 \sim 500 \mathrm{~nm}$ 
nanometer wax villi on the mastoid. Water droplets in each direction is free rolling on the surface, it is easy to assemble a big drop, so super hydrophobic characteristic of lotus leaf surface with isotropic and strong catchment characteristics.

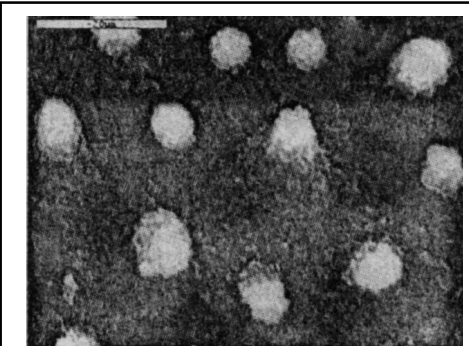

(a)Fresh lotus leaf $\times 2000$

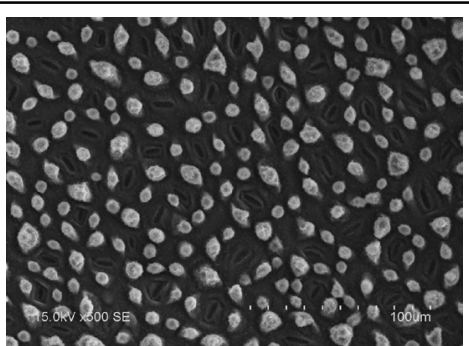

(b) Dried lotus leaf $\times 500$

Fig. 2. Lotus leaf SEM contrast photos

\subsection{Rice leaf}

The spreading of water droplets on the leaf surface of rice is shown in Fig. 3 Whether fresh or dried leaves, the water droplets are round and show better hydrophobic properties. Through the observation of rice leaf surface SEM diagram (refer with: Fig. 3), rice leaf surface micro-nano hierarchical structure is similar to the lotus leaf, by the diameter of micron 5 8 micro convex body and its diameter is about $20 \sim 50 \mathrm{~nm}$ acicular wax granules composed of two element composite structure, but in the rice surface, micron mastoid in parallel with the leaf edge direction (arrow), orderly arranged in the vertical direction of leaf edge are arranged disorderly. The angle between rice leaves and water was $142^{\circ}$, showing good hydrophobic and super hydrophobic characteristics. Rice leaves above the water droplets can roll along the direction parallel to the edges of the leaves of freedom, the direction of the rolling angle $\left(3 \sim 5^{\circ}\right)$ than the rolling edge of the vertical leaf angle $\left(9 \sim 15^{\circ}\right)$, expressed as hydrophobic properties anisotropy [6].

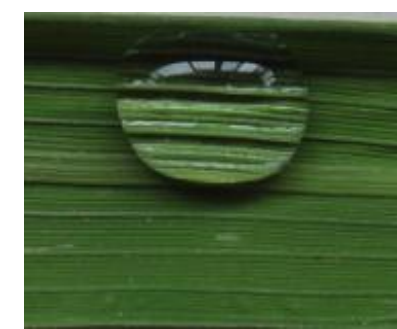

(a)Fresh leaf

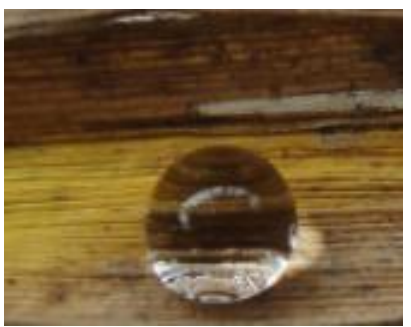

(b)Dried leaf

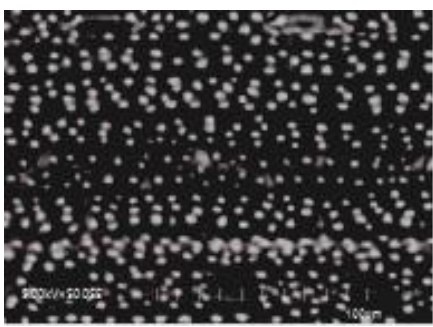

(c)SEM photo

Fig. 3. Wetting of water droplets on rice leaf samples

\subsection{Nasturtium}

Nasturtium is the rare wild plants of ranunculaceae. Its leaves are made up of many small hills high in 10 15um, as shown in Fig. 4(c). The hills are distributed on the surface of $100 \sim 150 \mathrm{~nm}$ nanometer scale clusters, $50 \mathrm{~nm}$ width nano column pile up. Thus, micro-nano structure ordered. The contact angle of nasturtium leaf is $145^{\circ}$, super hydrophobic.

With the increase of dehydration degree on the blade surface, the static contact angle has also changed, and the experimental results are shown in Fig. 4(d). With distilled water as a test fluid, the changing is shown in Fig. 5. 


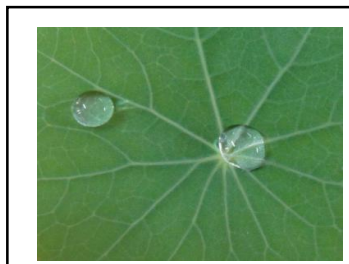

(a)Fresh leaf

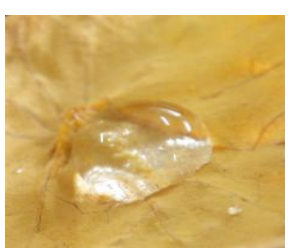

(b)Dry leaf

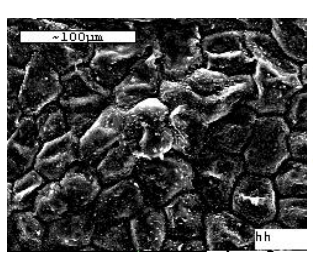

(c)SEM photo

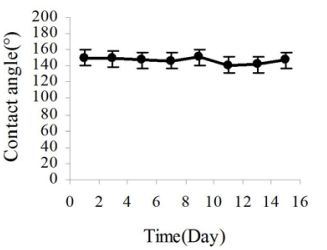

(d) Contact angle

Fig. 4. Nasturtium leaf wetting condition

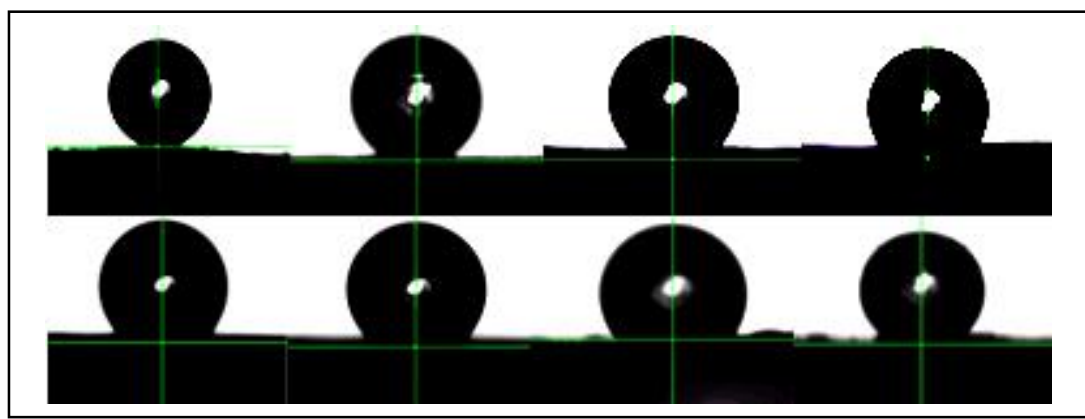

Fig. 5. Contact angle changing during drying

\subsection{Wettability comparison and analysis}

Barthlott and Neinhuist [3] have revealed for the first time that the superhydrophobic nature of lotus leaf surface is the result of the combination of micro- nano structure on its surface. On the basis of this research, Jiang Lei pointed out that the roughness caused by the micronano structure of the lotus leaf surface is the fundamental reason for the super hydrophobic and self-cleaning of lotus leaves. From the water droplets in the leaf surface spreading, water contact angle measurements and SEM observation etc. we found that several plant leaf scales have good hydrophobic or super hydrophobic properties, they have micro-nano composite microstructure that is because they are so with micro-nano morphology, which is the main reason of hydrophobic or super hydrophobic properties of typical plant leaves.

The leaves of these three kinds of plants have different wettability. The lotus leaves and nasturtium leaves are isotropic, while the leaves of rice are anisotropic.

\section{Study on biomimetic application of aluminum alloy materials}

It is found that the micro-nano composite structure has important reference value for the preparation of biomimetic superhydrophobic surface by studying the leaf table of superhydrophobic plants[7,8]. By combining chemical etching with surface modification, the micro - nano composite structure on the surface of aluminum alloy has been successfully produced, which makes it appear super hydrophobic $[9,10]$.

\subsection{Surface modification of aluminum alloy by chemical etching}

Aluminum Alloy is divided into rectangular pieces of $2 \times 2.5(\mathrm{~cm})$, polished with fine sandpaper, make it look very smooth, followed by acetone soaking aluminum 
decontamination, deionized water ultrasonic cleaning, and then immersed into $10 \mathrm{~min}$ hydrofluoric acid solution of $3.75 \mathrm{~mol} / \mathrm{L}$ to remove the surface oxide layer.

The chemical etching solution was prepared with $\mathrm{HC} 1, \mathrm{HNO}$ and $\mathrm{HF}$ with volume ratio of 15:5:1, and the aluminum sheet was soaked in corrosive solution at room temperature for chemical etching. When corrosion, gently clamp the surface of aluminum sheet with tweezers, turn over in the corrosive liquid, and make a large amount of gas overflow. After the reaction is complete, the aluminum sheet is quickly flushed with a large amount of water, and the surface is scrubbed with absorbent cotton. After drying, the rough micro / nano structure with two different scales is prepared[11].

\subsection{Modification of micro nano structured surface with Fas}

It is not enough to construct micro / nano composite structures on the surface of metal substrate. After using the low surface energy material to modify the micro nano structured metal surface, it has both micro nano structure and very low surface energy.

At room temperature, Fas and water are mixed at a volume ratio of $1: 49$ to form a $2 \%$ intermediate solution of Fas. Then, the $\mathrm{PH}$ value of the intermediate solution is adjusted between 3 and 3.8 by glacial acetic acid. The amount of adding Fas to dilute ethanol, arranged in 1\% (WT) fluoroalkyl alcohol solution, stirring for about 5 hours at the $50 \mathrm{r} / \mathrm{min}$ rate in a magnetic stirrer. After forming a homogeneous and transparent solution, the Fas solution is placed to be used[12].

After the chemical etching, the surface of the aluminum sheet has hydrophobic characteristics, but the surface is hydroxylated. The aluminum fluoride soaked in Fas solution for $30 \mathrm{~min}$, the hydroxylated surface reacts with the silane hydrolysis group, forming a layer of nano FAS-SAM, the final sample placed one or two days to be dried, combined with Fas nonstick film is completely formed.

\subsection{Test results}

The Fig.6 is the SEM photos of aluminum alloy surface etched by strong acid solution for 12 min after being zoomed 3000 magnification, contact angles photos of water droplets on superhydrophobic aluminum alloy surface are also visible. On the surface of the etched aluminum alloy, the labyrinth structure are formed by consists of many rectangular bosses and pits. A micronscale smaller boss is also distributed on the nanoscale boss. There are many nano scale bump on micro scale convex platform, and these micro and nano convex layers are stacked into ladder like distribution. This shows that the super hydrophobic aluminum alloy surface has micron and nano scale double roughness structure. The contact angle is $156^{\circ}$. It shows that it has very good super hydrophobic characteristics.

In addition, the static contact angle data of the superhydrophobic aluminum alloy surface were not changed obviously after 6 months in the air. This shows that the surface of the superhydrophobic aluminum alloy prepared in the experiment has good time stability. 


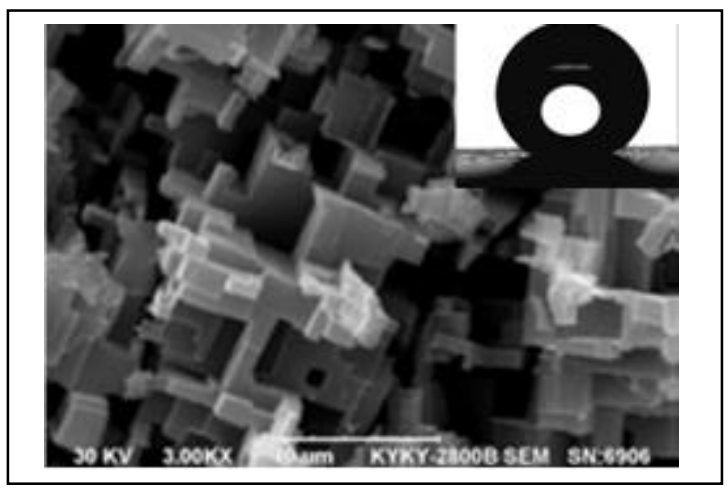

Fig. 6. EM photos of etched aluminum alloy surface

\section{Conclusions}

Through the measurement of static contact angle, scanning and data processing, the positive and negative wettability of lotus leaves was studied. The results showed that the degree of dehydration had little influence on wettability, and hydrophobicity did not change.

A comparative study of the hydrophobic mechanism shows that the hydrophobic mechanism of lotus, nasturtium and rice leaves is because of their micro-nano composite structure. Both the lotus leaves and nasturtium leaves have isotropic wetting properties, while the leaves of rice are anisotropic, i.e., in parallel with the blade edge, the hydrophobicity is stronger than that perpendicular to the blade edge.

Modeled on the surface of lotus leaves and nasturtium super hydrophobic and hydrophobic, the surface of aluminum alloy is chemical etched and low surface energy material modified to form micro-nano ladder rough structure of bosses and pits. Its contact angle is $156^{\circ}$ showing good super hydrophobic characteristics.

\section{References}

1. L. Feng, H. S. Li, L. Jiang. Adv. Mater, 24, 14 (2002), p1857

2. C. Neinhuis, W. Barthlott. Annals of Botany, 79,6 (1977), p.667

3. W. Barthlott, C. Neinhuis. Planta , 202, 1 (1997)1, p.1

4. A. B. Cassie, S. Baxter. Trans Faraday Soc, 40, 8 (1944), p546

5. A. Lafurna, D. Quere. Nature Mater, 7, 2 (2003)2, p.457

6. X. F. Zhao, M. Q. Yang, L. Zhang. Chemical Industry and Engineering Process, 35, 9 (2016), p.2812(In Chinese)

7. A. Terray, J. Oakey, D. W. Marr. Science, 5574, 296 (2002), p.1841

8. A. D. Tserepi, M. E. Vlachopoulou, E. Gogolides. Nanotechnology, 17, 15 (2006), p.3977

9. M. H. Sun, C. X. Luo, L. P. Xu. Langmuir, 19, 21 (2005) 1, p 8978(In Chinese)

10. J.L. Song. Study on surface preparation and application of extreme wettability of engineering metal materials (Ph.D., Dalian University of Technology, China 2015), p.42 (In Chinese)

11. A . Nakajima, D. Fujishima, K. Hashimoto. Adv Mater, 16, 11 (1999)1, p.1365

12. R. N. Wenzel. Industrial and Engineering Chemistry, 28, 8 (1936), p.988 